\title{
UNIFIED SCHEME FOR SEYFERTS OR THE INFLUENCE OF INTERACTIONS?
}

\author{
D. DULTZIN-HACYAN \\ Instituto de Astronomía UNAM, México \\ IVETTE FUENTES GURIDI AND YAIR KRONGOLD \\ Facultad de Ciencias, UNAM, México \\ AND \\ PAOLO MARZIANI \\ Osservatorio Astronomico de Padova, Italia
}

\section{Introduction}

The first statistical study of the environment of Seyfert galaxies (of both types 1 and 2) was done by Petrosian (1982), who found that Sy2 galaxies had an excess of nearby companion galaxies with respect to field galaxies. Dahari (1985) made a similar study, and obtained that both types of Seyfert galaxies are found five times more often in interacting systems that "normal" galaxies. Fuentes-Williams \& Stocke (1988) obtained the opposite result: that Seyfert galaxies have no significative excess of nearby companions, and MacKenty (1989) obtained the same result as Petrosian. The last papers appeared in 1995: Rafanelli, Violato \& Baruffolo (1995) obtained a similar result as Dahari. Laurikainen \& Salo (1995) made a careful attempt to understand the discrepancies; they concluded that the incompatibilities in the results were mainly due to control sample selection, and problems in background galaxy determination. Their work confirmed the result of the first study. However, in order to explain the discrepancies between previous results, they also used incorrectly defined control samples. Objections can be made against all of these works for this only reason. We have repeated the study using, for the first time, the digitized Palomar Sky Survey plates (POSS), an automated selection procedure, and correctly defined control samples (Osterbrock 1993). 


\section{Methodological improvements to study the environment of Seyfet galaxies and results}

The sample of Seyfert galaxies was compiled from Véron-Cetty \& Véron (1991). It consists of $72 \mathrm{Sy} 1$ and $60 \mathrm{Sy} 2$. Both samples are volume limited, and the $V / V_{\max }$ test assures completeness to a level of $92 \%$. The redshifts are limited to $0.007 \leq z \leq 0.035$, and we selected galaxies with galactic latitudes $b \geq 45^{\circ}$, in order to avoid extinction, and confusion due to galactic stars. Rich clusters were avoided.

For the control sample, the above criteria were also imposed. In this work we used complete samples for the first time, but an even more important methodological improvement is the definition of control samples of field galaxies which match the Seyfert galaxies in all respects except that they are not Seyfert galaxies. In oreder to achieve this, two control samples were defined, one for each type of Seyfert galaxies, because both the Hubble type and redshift distributions of the two types of Seyfert galaxies differ, and our aim was to match these distributions. The control samples were obtained by a process of multiple random extraction from more than 10,000 objects of the CfA catalog (Huchra et al. 1983). For each control sample we matched Hubble type, and redshift distributions. We did not match absolute magnitudes, since this would introduce instead of eliminate a bias (Seyfert galaxies are brighter due to the nucleus), instead we matched the diameters distribution. The control samples are complete (in volume) to a confidence level of up to $97 \%$. Although the above mentioned similarities were long ago known to be required for a proper comparison, in previous works matching the distributions was impossible maintaining the same densities, due to the selection of small control samples from nearby galaxies. In this work the background densities between samples are statistically equal (we applied Mann-Whitney's U test). Other selection details, histograms, and statical tests will be given in forthcoming paper.

In order to look for companions, we analyzed more than 500 digitized POSS plates using the automated detection package FOCAS (Faint Object Classification and Analysis System; Jarvis \& Tyson 1981). The correct use of FOCAS requires a fine tunning of several parameters which shall be discussed elsewhere. This procedure reduces to a minimum the subjective bias present in all previous works, which were done counting galaxies from the POSS prints "by eye". The search for companions was done in circular areas around each galaxy, with radii equal to three times the diameter of the galaxy. Obviously, some of the neighbors are background galaxies. The correct estimation of the background galaxy density is crucial. As in previous studies, we assumed a Poisson distribution of background galaxies, but in this work, the determination of the number density $\rho$, was made directly from the digitized POSS plates in regions of one sqaure degree surrounding 
each galaxy. The use of the Lick counts given by Shane \& Wirtanen (1967) to estimate the projection effects can introduce an important bias (as in Rafanelli et al. 1995). The final result we obtained is a confirmation of the first result obtained by Petrosian (1982): It is only Sy2 galaxies that have excess companions, but not Sy1 galaxies. The excess factor for Sy2 with respect to their control sample is 1.8 (less than the value of 2.7 obtained by Laurikainen \& Salo in 1995). Our result is relevant to a confidence level of $99.5 \%$ according to a chi square test. Other statistical test were also applied, but they will be discussed elsewhere.

\section{Interpretation of the results}

Two types of Seyfert galaxies were defined by Khachikian \& Weedman (1971) on the basis of spectroscopic properties. Miller \& Goodrich (1990) discovered that in plane-polarized light some Sy2 have the spectrum of a Sy1. The interpretation they proposed was that those galaxies have a Broad Line Region (BLR), whose radiation does not escape directly because of strong obscuration by a dusty torus around it, but which does escape along the axis of the torus, and is scattered towards us (and thus polarized) by free electrons above and below the obscuring torus. The extrapolation implying that all Sy2 galaxies are obscured Sy1 galaxies, oriented in such a way with respect to the observer, that the BRL is hidden is not so obvious, and yet this has become an almost unquestionable paradigm, known as the "Unified Scheeme for Seyferts". We want to stress that Miller \& Goodrich (1990) clearly stated that many bright Sy2 observed by them do not show the broad emission lines (see also Villeux, Sanders \& Kim 1997).There are many other indications of intrinsic differences between Sy1 and Sy2 galaxies. Just to mention a few: Heckman et al. (1989) discovered that Sy2 have larger $L_{\mathrm{CO}} / L_{\mathrm{B}}$ ratios than Sy1. Dultzin-Hacyan, Masegosa \& Moles (1990) showed that while the mid-infrared $(\sim 25 \mu \mathrm{m})$ emission in Sy1 is synchrotron radiation (or dust re-emission of it), in Sy2 it is dust re-emission of starlight (see also Mouri \& Taniguchi 1992; Mouri, Kawara \& Taniguchi 1997). Norris \& Roy (1997) showed that Sy2 (and not Sy1) follow the FIR vs radio correlation, as Starburst (SB) galaxies. Even in the X-rays, Sy2 have similar properties as SB galaxies (Muzhotsky 1997), except for the very few cases where the $\mathrm{FeK}_{\alpha}$ line is found (these may be indeed obscured Sy1 nuclei).

An alternative to the unified scheeme was proposed to explain the different properties of the two types of Seyfert galaxies in Dultzin-Hacyan (1995). In this new scheeme, radiation due to accretion unto a black hole decreases, while the relative contribution of a circumnuclear starburst radiation increases, from Seyfert nuclei types 1 to 2 . This scheeme is supported 
by the above mentioned observations and by statistical studies of the multifrequency emission of Seyferts (Mass-Hesse et al. 1995; Dultzin-Hacyan \& Ruano 1996). It is also clearly supported by this work, because the difference in environment disagrees with the interpretation that the two types of Syferts represent similar objects seen from different viewing angles. Further discussion and interpretation of our result will be given in a forthcoming paper.

\section{References}

Dahari, O. 1985, ApJS, 57, 643

Dultzin-Hacyan, D., Masegosa, J. \& Moles, M. 1990, A\&A, 238, 28

Dultzin-Hacyan, D. 1995, RMA\&A SC 3, 31

Dultzin-Hacyan,D. \& Ruano, C.1996, A\&A, 305, 719

Fuentes-Williams, T. \& Stocke, J.T. 1988, AJ, 93, 1235

Huchra, J., Davis, M., Latham, D. \& Tonri, J. 1983, ApJS 52, 89

Jarvis, B.J. \& Tyson, H. 1981, AJ, 86, 476

Khachikian E.Ye. \& Weedman D.W. 1971, Astrofizika, 7, 389

Laurikainen,E. \& Salo, H. 1995, A\&A, 293, 683

MacKenty, J.W. 1989, ApJ, 343, 125

Mass-Hesse, J.M., Rodríguez-Pascual, P.M., Sanz Fernández de Córdoba, L, Mirabel, I.F., Wamsteker, W., Makino, F. \& Otani, C. 1995, A\&A, 298, 22

Miller, J.S. \& Goodrich, R.W. 1990, ApJ, 355, 456

Mouri, H. Kawara, K. \& Taniguchi, Y. 1997, ApJ, 484, 222

Mouri, H. \& Taniguchi, Y. 1992, ApJ 386, 68

Muzhotsky, R. 1997, RMA\&A SC 6, 213

Norris, R.P. \& Roy, A.L. 1997, RMA\&A SC 6, 230

Osterbrock, D.E. 1993, ApJ, 404, 551

Petrosian, A.R. 1982, Astrofizika, 18, 548

Rafanelli, P., Violato, M. \& Baruffolo, A. 1995, AJ, 109, 1546

Shane, C.D. \& Wirtanen, C.A. 1967, Publ. Lick Obs., 22, 647

Véron-Cetty, M.P. \& Véron, P. 1991, A Catalogue of Quasars and Active Nuclei, 5th ed. (ESO, Garching)

Villeux S., Sanders D. B. \& Kim D.-C. 1997, ApJ 484, 92 